\title{
Plasmon resonance optical tuning based on photosensitive composite structures
}

\author{
Gilardi, Giovanni; Xiao, Sanshui ; Mortensen, N. Asger; d'Alessandro, Antonio; Beccherelli, Romeo
}

Published in:

Optical Society of America. Journal B: Optical Physics

Link to article, DOI:

10.1364/JOSAB.31.000360

Publication date:

2014

Document Version

Publisher's PDF, also known as Version of record

Link back to DTU Orbit

Citation (APA):

Gilardi, G., Xiao, S., Mortensen, N. A., d'Alessandro, A., \& Beccherelli, R. (2014). Plasmon resonance optical tuning based on photosensitive composite structures. Optical Society of America. Journal B: Optical Physics, 31(2), 360-365. https://doi.org/10.1364/JOSAB.31.000360

\section{General rights}

Copyright and moral rights for the publications made accessible in the public portal are retained by the authors and/or other copyright owners and it is a condition of accessing publications that users recognise and abide by the legal requirements associated with these rights.

- Users may download and print one copy of any publication from the public portal for the purpose of private study or research.

- You may not further distribute the material or use it for any profit-making activity or commercial gain

- You may freely distribute the URL identifying the publication in the public portal 


\title{
Plasmon resonance optical tuning based on photosensitive composite structures
}

\author{
Giovanni Gilardi, ${ }^{1}$ Sanshui Xiao, ${ }^{2}$ N. Asger Mortensen, ${ }^{2}$ Antonio d'Alessandro, ${ }^{3}$ and Romeo Beccherelli ${ }^{4, *}$ \\ ${ }^{1}$ COBRA Research Institute, Photonic Integration Group, Department of Electrical Engineering, \\ University of Technology Eindhoven, The Netherlands \\ ${ }^{2}$ DTU Fotonik, Department of Photonic Engineering, Technical University of Denmark, \\ DK-2800 Kongens Lyngby, Denmark \\ ${ }^{3}$ Dipartimento di Ingegneria dell'informazione, elettronica e telecomunicazioni, \\ Sapienza Universitá di Roma, Via Eudossiana 18, 00184 Rome, Italy \\ ${ }^{4}$ Consiglio Nazionale delle Ricerche-Istituto per la Microelettronica e Microsistemi (CNR-IMM), \\ Via del fosso del cavaliere 100, 00133 Rome, Italy \\ *Corresponding author: romeo.beccherelli@artov.imm.cnr.it
}

Received September 5, 2013; revised November 26, 2013; accepted December 8, 2013; posted December 19, 2013 (Doc. ID 194727); published January 29, 2014

\begin{abstract}
This paper reports a numerical investigation of a periodic metallic structure sandwiched between two quartz plates. The volume comprised between the quartz plates and the metallic structure is infiltrated by a mixture of azo-dye-doped liquid crystal. The exposure to a low power visible light beam modifies the azo dye molecular configuration, thus allowing the wavelength shift of the resonance of the system. The wavelength shift depends on the geometry of the periodic structure and it also depends on the intensity of the visible light beam. (C) 2014 Optical Society of America

OCIS codes: (160.3710) Liquid crystals; (240.6680) Surface plasmons; (230.3720) Liquid-crystal devices. http://dx.doi.org/10.1364/JOSAB.31.000360
\end{abstract}

\section{INTRODUCTION}

In 1997 Ebbsen et al. demonstrated how making a periodic arrangement of subwavelength holes in an otherwise optically opaque metal film may induce an extraordinary amount of light transmittance for certain frequencies, the so-called extraordinary optical transmission [1]. The enhanced transmittance is attributed to the excitation of surface-plasmon polaritons (SPPs) [2]. The transmission of light can be totally suppressed by a regular periodic arrangement of subwavelength structures [3]. It is attributed to the sign change at the metal/dielectric interface of the real part of the dielectric permittivity of the metal evanescent SPPs fields that are highly confined at the boundary of the metal. The manipulation of plasmon resonances in metallic nanostructures has been attracting great interest due to their widely anticipated applications in microscopy [4] , waveguides [5], and lasers [6]. To improve the performance of plasmonic devices, significant effort has been devoted toward optimizing the quality of SPPs as well as their tunability.

Tuning the surface-plasmon resonance (SPR) of metallic nanostructures has been the subject of many recent studies, both experimentally and theoretically [글 $]$. The possibility of tuning the resonance of metallic structures is of particular interest in many applications as SPR biosensing instruments in the determination of affinity parameters for biomolecular interactions [10], optofluidic sensors [11], and absorbers with extended working frequency ranges $[\underline{12}, 13]$. Two approaches are generally investigated: (1) the tuning based on the modification of the geometric parameters [14] and (2) the tuning based on the modification of the medium surrounding the plasmon structure [15]. For the geometric tuning, when the period of the geometry and the thickness of the metal film are varied, the resonant wavelength changes slightly [15]. The resonant wavelength is redshifted when increasing the period, while it is blueshifted when increasing the thickness of the metallic film [16]. The variation of the surrounding's dielectric properties is a much more effective method [15], because it allows a real time, continuous, and reversible tuning of the plasmonic resonance. Liquid crystals (LC) have proven to be effective in tuning optical properties of plasmonic devices [17-23]. A structure based on gold nanodisk plasmonic crystal controlled by dual frequency liquid crystal was presented in $[18,19]$. By adding an azo dye to the LC, the concept was extended to all-optical control of a hybrid system composed of photoswitchable gratings and $\mathrm{Au}$ identical nanodisk arrays [22], to a plasmonic absorber using a liquid-crystal-coated asymmetric nanodisk array by mixing the liquid crystal with and to an asymmetric nanodisk array [23].

In this paper, we investigate a novel composite structure proposing regular gold rings, rather than only disks, with a nematic liquid crystal (NLC) doped with an azo dye. We present the model used to evaluate the effects of the external optical pump and calculate the effects of the boundary conditions and geometrical parameters and shows the MR:LC refractive index distribution in the cell volume. Finally, we present the optical response that shows that an excess of structure behaves as a tunable absorber with an excess of $30 \mathrm{~dB}$ absorption that can be geometrically and optically tuned over a broad range of wavelengths. 


\section{DEVICE GEOMETRY AND PHYSICS}

Figure 1 illustrates the structure under investigation, which consists of a $2 \mathrm{D}$ array of gold rings on a quartz substrate, with a period $T$. The thickness, internal radius, and external radius of the rings are denoted with $h, a$, and $b$, respectively. The plasmon resonances in the near infrared spectrum region are found for $T=800 \mathrm{~nm}$ and $b=300 \mathrm{~nm}$ [15].

For $a=0$ the gold rings degenerate into gold disks. The ring arrays can be fabricated by using the standard electronbeam lithography, as discussed in [16]. The structure, as so far described, is covered with an upper quartz plate. The separation of the two quartz plates is $t=1000 \mathrm{~nm}$. This value can be achieved by means of silica ball spacers or photopatterned posts. After gluing and partially sealing the plates, we consider here that the volume comprised between the quartz plates and the gold rings is infiltrated by a mixture of azo-dyedoped LC.

In our simulations we will consider a mixture composed of the NLC E7 doped with methyl red (MR), $2 \%$ in weight, which is the highest possible soluble quantity of MR in NLC (MR:E7) to minimize the pump power required for optical tuning [24]. E7 and MR are easily available and have well-known optical properties that allow immediate comparison with previous works $[\underline{25}, \underline{26}]$. At $\lambda=1550 \mathrm{~nm}$, the refractive indices of $\mathrm{E} 7$ are $n_{\text {par }}=$ 1.689 and $n_{\text {perp }}=1.5$ for light polarization parallel and perpendicular to the LC molecular director, $n$, respectively.

At thermal equilibrium, with no applied external stimulus, $\mathrm{MR}$ is in its elongated trans form. The MR:E7 mixture behaves as a guest-host optically anisotropic mixture of an LC compound and a nonliquid crystalline compound. The small quantity of MR does not significantly modify the refractive index of the mixture with respect to the pure E7. In this mixture, the long axis of the LC molecules and the long axis of trans-MR remain aligned parallel to the $z$ axis. This prealignment of the MR:E7 may be induced by a few nanometers thick layer of polymer Nylon 6 (not shown in Fig. 1. deposited on the surface of both quartz plates and properly rubbed along the $z$ direction, which is neither affected by the pump power nor considered explicitly in the numerical model. Methyl red turns in the spherical cis form when the structure is irradiated by a visible TE-polarized (electrical field parallel to $z$ ) pump laser at the wavelength $\lambda=532 \mathrm{~nm}$, which is in the absorption band of MR. Methyl red conformational molecular transition brakes
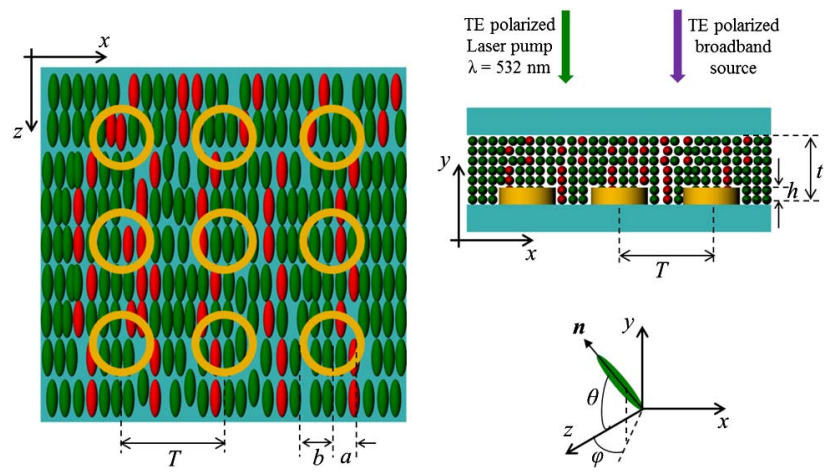

Fig. 1. Schematic of the device. $T$ is the period of the structure, $a$ and $b$ denote the internal and external radius of the rings, respectively, $h$ is the thickness of the rings, and $t$ is the distance between the quartz plates. The bottom left inset sketches the orientation of the liquid crystal molecular director, $n$. the directional order of the MR:E7 guest host. In this condition, the MR:E7 mixture behaves like an optically isotropic material since no preferential orientation is induced to the LC. The local refractive index of the mixture after the exposure can be estimated as the average value given by [27]

$$
\langle n\rangle=\sqrt{\frac{n_{\text {par }}^{2}+2 n_{\text {perp }}^{2}}{3}}=1.565 .
$$

Upon reduction of the pump power, nematic order is recovered, due to the interaction with the alignment layer. By continuously changing the power of the optical pump, it is possible to vary the refractive index of the mixture seen by an impinging TE-polarized light beam (electric field parallel to the $z$ direction) from 1.5 to 1.565 .

The working principle of the proposed device is based on the variation of the refractive index of the MR:E7 mixture by applying an external optical pump in the visible spectrum. In detail, we evaluate the plasmon resonance shift in relation to the optical power of the pump laser.

\section{MODELING OF THE MIXTURE ORIENTATION}

The orientation of the NLC director distribution is computed by minimizing the MR:E7 free energy by solving the partial Euler-Lagrange equation in the weak form. We use the finite element method implemented in a commercial solver [28,29]. The free energy of the MR:E7 mixture takes into account the elastic energy of the LC and the molecular reorientation induced by the deformation of the exposed MR [30],

$$
F=F_{\mathrm{LC}}+F_{\mathrm{MR}},
$$

where

$$
\begin{aligned}
F_{\mathrm{LC}}= & \frac{1}{2} K_{11}(\nabla \cdot n)^{2}+\frac{1}{2} K_{22}[n \cdot(\nabla \times n)]^{2} \\
& +\frac{1}{2} K_{33}[n \times(\nabla \times n)]^{2} \\
F_{\mathrm{MR}}=- & \frac{1}{2} \eta \varepsilon_{0}\left[\Delta \varepsilon_{M R}\left(n \cdot \vec{E}_{\mathrm{PUMP}}\right)^{2}+\varepsilon_{M R} \vec{E}_{\mathrm{PUMP}} \cdot \vec{E}_{\mathrm{PUMP}}\right] .
\end{aligned}
$$

Here, $F_{\mathrm{LC}}$ depends on the elastic constant of the LC, while $F_{\mathrm{MR}}$ is related to the intensity of the visible pump laser. The values considered in Eqs. (3) and (4) are: $K_{11}=12 \mathrm{pN}, K_{22}=$ $7.3 \mathrm{pN}$ and $K_{33}=17 \mathrm{pN}$. $\eta$ is the diffraction efficiency as defined in [31]. The boundary conditions imposed by the mixture/quartz surfaces play a role in the evaluation of the total energy of the mixture. In our model we consider the LC molecules aligned in the z-direction $(\varphi=0)$ and the molecular director parallel to the plane $x z(\theta=0)$. Strong boundary conditions are assumed as typical of rubbed nylon. By solving Eq. (2), it is then possible to calculate the local refractive index of the mixture as a function of the pump power.

Figure $\underline{2}$ shows the refractive index distribution when the power of the optical pump is changed. It clearly identifies a bulk reorientation of the mixture. There is not a relevant reorientation of the mixture near the mixture/quartz interfaces, due to strong anchoring conditions in those regions. The refractive index change occurs from the vertical center of the structure (about $y=500 \mathrm{~nm}$ ), where the effect of the 
boundary conditions is weak, and then propagates to the interfaces. The refractive index value is almost independent of the $x$ coordinate, while it is strongly dependent on the $y$ coordinate. Furthermore, the refractive index distribution does not depend too much on the $a$ parameter. In other words, there are no significant differences between disks and rings as far as the refractive index profile is concerned, as Fig. 2 clearly shows.

Figure 3 reports the refractive indices of the MR:E7 mixture as a function of laser power at three specific points of the cell. The point considered are $P_{1}=\left(x_{1}, y_{1}\right)=(0,500 \mathrm{~nm})$, $P_{2}=\left(x_{2}, y_{2}\right)=(0,750 \mathrm{~nm})$, and $P_{3}=\left(x_{3}, y_{3}\right)=(0,900 \mathrm{~nm})$, as reported in Fig. 2. A similar behavior for the refractive index of the mixture is found, for the same value of gold thickness $(h=20 \mathrm{~nm})$ and for a disk with $a=250 \mu \mathrm{m}$. This is a natural consequence of the reasonable assumption that pump power is substantially uniform with respect to $x$ and $y$.

The comparison among $P_{1}, P_{2}$, and $P_{3}$ shows that the reorientation of the mixture occurs for lower optical pump values at the point close to the vertical center of the structure. Again, that is related to the weaker effect of the imposed strong boundary conditions further away from the surfaces and to the assumption that pump power is substantially uniform with respect to $\mathrm{x}$ and $\mathrm{y}$. From Fig. $\underline{3}$, it is also possible to define a threshold laser pump $\left(P_{\mathrm{th}}\right)$ and a saturation laser pump $\left(P_{\mathrm{sat}}\right)$. $P_{\text {th }}$ is the particular value of the laser pump where the elastic forces of the LC and the mechanical forces of the MR are equal. If the laser pump power is below $P_{\text {th }}$, there is no reorientation of the MR:LC mixture because the MR does not have enough strength to disrupt the LC order.

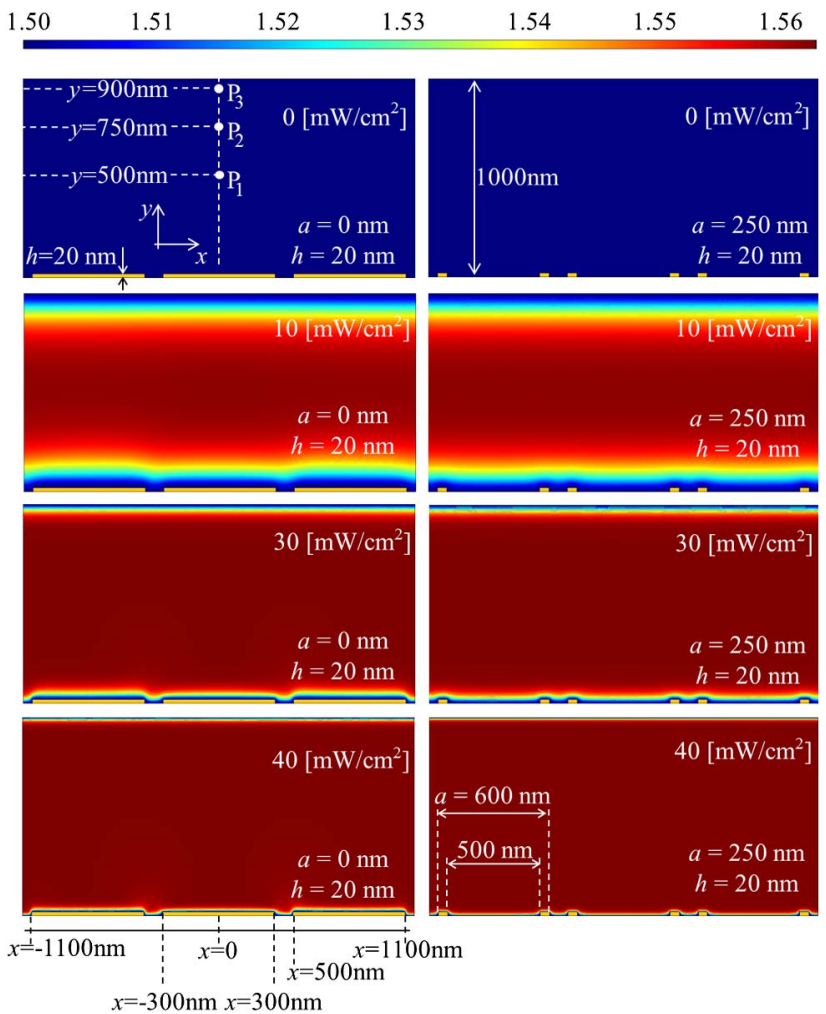

Fig. 2. Mixture refractive indices for four values of the optical pump. Refractive index variation for disk ( $a=0 \mathrm{~nm}$ ) (left) and ring with $a=$ $250 \mathrm{~nm}$ (right). For all cases reported, $T=800 \mathrm{~nm}, b=300 \mathrm{~nm}$, $h=20 \mathrm{~nm}$, and $t=1000 \mathrm{~nm}$.

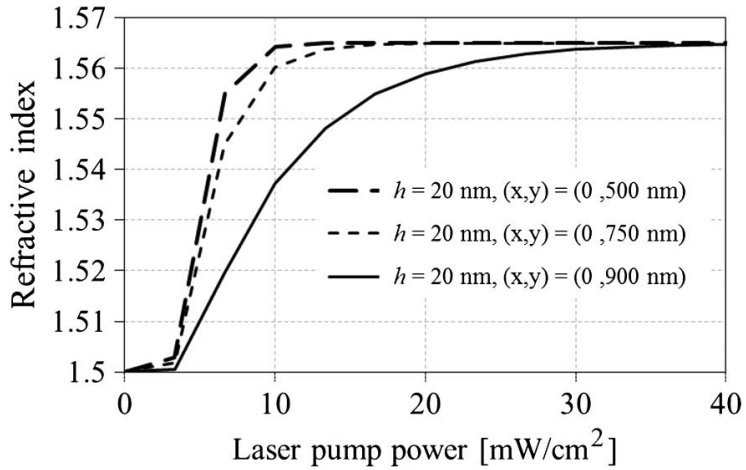

Fig. 3. MR:E7 mixture refractive index for three different points of the cell versus laser pump power. The geometrical parameters considered are $T=800 \mathrm{~nm}, a=0, b=300 \mathrm{~nm}$, and $t=1000 \mathrm{~nm}$.

The reduction of the MR:E7 mixture order starts when the pump power overcomes $P_{\text {th }}$. The reorientation of the LC, and then the changing of the refractive index, occurs until the pump power reaches $P_{\text {sat }}$. We define $P_{\text {sat }}$ as the pump power to apply to completely transform the MR. According to our simulations, the value of $P_{\text {sat }}$ is about $40 \mathrm{~mW} / \mathrm{cm}^{2}$. The value obtained for $P_{\text {sat }}$, matches the experimental results reported in [32] in which a similar volume of mixture was used and where the anchoring was associated with similar boundary conditions.

\section{SPECTRAL RESPONSE}

After solving the problem of the refractive index distribution, we investigate the relation between the pump power and the spectral response of our device in the near infrared telecom window.

All spectra are computed by considering a free space beam generated by a broadband source impinging with normal incidence on the structure, i.e., propagating along the $-y$ axis. The problem is studied for the TE-polarized light (electric field parallel to $z$ ). We consider the periodic boundary conditions in the $x$ and $z$ directions, and the absorbing boundary condition in the $y$ direction. Dispersive properties of gold are taken into account using the experimental data reported in [33] while the refractive index considered for the quartz plates is fixed as 1.44 when the wavelength is $1550 \mathrm{~nm}$.

Figure 4 shows the effect of the variation of $h$, when $a=0$ and without any optical pump. The resonance is blueshifted when the thickness of the gold disk is increased. We note that the SPP modes supported by the two interfaces strongly couple with each other in the thin-film limit, while they are almost decoupled for a thicker film, i.e., $h=150 \mathrm{~nm}$. Under this circumstance, the SPP resonance is related only to the mode for the single interface.

The SPP resonance appears when the plasmon wave vector matches the wave vector of the incident photons and gratings as in [17],

$$
k_{\mathrm{SPR}}=k_{\|} \pm g P_{x} \pm f P_{y},
$$

where $k_{\mathrm{SPR}}$ is the surface-plasmon wave vector, $k_{\|}$is the component of the incident light wave vector in the plane of the grating, and $P_{x}=P_{y}=2 \pi / T$ is the reciprocal vector of the grating for the square array. In all computations, the depth of the suppressed wavelength of the transmitted signal 


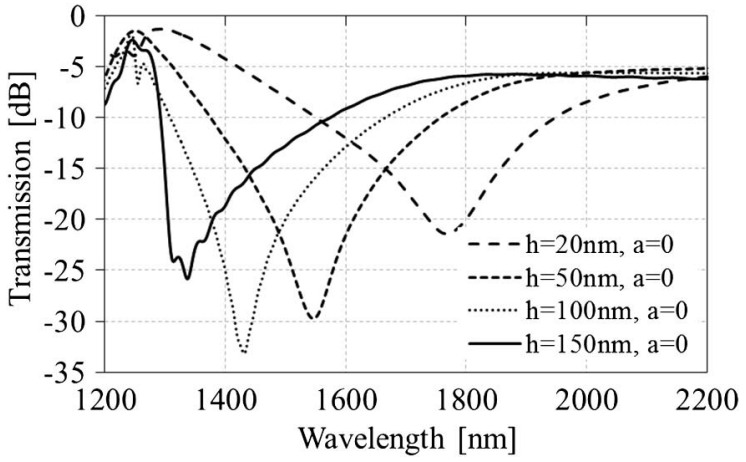

Fig. 4. Simulated zero-order transmittance spectra as a function of the gold thickness, for $a=0 \mathrm{~nm}, b=300 \mathrm{~nm}$, and $T=800 \mathrm{~nm}$, without a pump signal.

exceeds $20 \mathrm{~dB}$. The behavior of the spectral responses at around $1250 \mathrm{~nm}$ is due to the Rayleigh anomaly defined by $\lambda_{R}=n_{\text {quartz }} \cdot T$ and it does not depend on $h$. In an effectiveindex picture, the variation of $h$ and a induces a change in the SPP dispersion relation and its effective index.

Figure 5, in addition to Fig. 4, describes the tuning of the spectral response when the internal radius and the thickness of the rings changes. The curves in Fig. $\underline{5}$ are well fitted by quadratic curves, indicating a linear dependence on the gold-surface coverage. The case $a=0 \mathrm{~nm}$ corresponds to the resonant wavelength position reported in Fig. 4 .

Next, we investigate the effect of the laser pump. Figure 6 shows the transmittance when the device, based on periodic disks $(a=0 \mathrm{~nm})$, is exposed to a pump laser beam of $40 \mathrm{~mW} / \mathrm{cm}^{2}\left(P_{\text {sat }}\right)$, which induces a complete disruption of the order of mixture MR:E7, compared to the case without optical pumping.

The application of the pump implies the increase of the refractive index of the structure for the defined TE polarization.

This leads to the increase of corresponding $k_{\mathrm{SPR}}$, which induces redshift of the plasmon resonance. The tuning obtained in the configurations proposed in Fig. 6 decreases from 40.8 to $29 \mathrm{~nm}$ when $h$ increases from 20 to $150 \mathrm{~nm}$, but the relative wavelength shift remains substantially unchanged at $2.3 \pm 0.2 \%$. Besides, we show in Fig. 7 the effect of the optical pump, in the case of $a=250 \mathrm{~nm}$ (rings). Note that, when having the same periodicity, the resonant wavelength for the disks array is quite different from that for the ring structure. This is mainly due to the different SPP dispersion arising from the coupling of SPP modes. Note that the phase matched condition, see Eq. (5), is fulfilled for both cases.

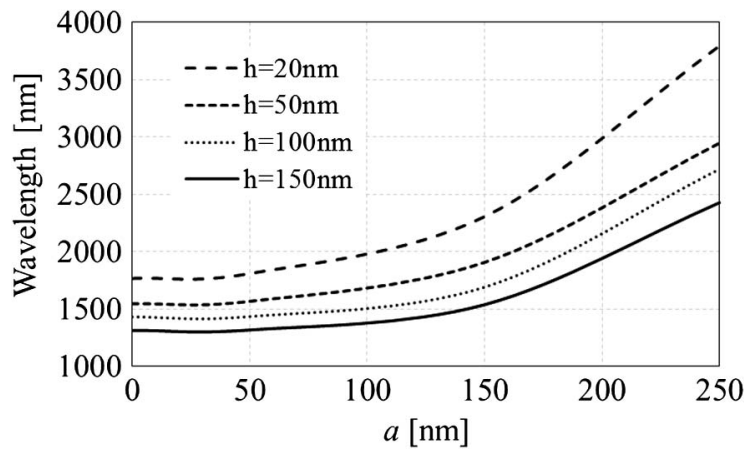

Fig. 5. Resonant wavelength position as a function of the gold thickness, for $b=300 \mathrm{~nm}$ and $T=800 \mathrm{~nm}$, without a pump signal.

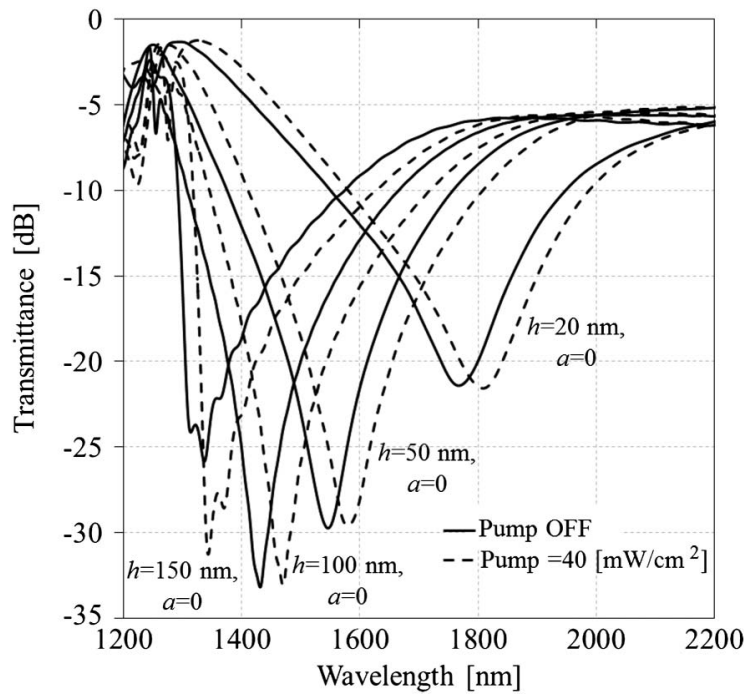

Fig. 6. Simulated zero-order transmittance spectra as a function of the gold thickness $(h)$. The geometry considered is $a=0 \mathrm{~nm}$ (disks), $b=300 \mathrm{~nm}$, and $T=800 \mathrm{~nm}$. The dashed and solid lines compare optical pumping with $P_{\mathrm{sat}}=40 \mathrm{~mW} / \mathrm{cm}^{2}$ to the case of no pumping.

The tuning obtained for the configurations simulated in Fig. 7 decreases from 122 to $45 \mathrm{~nm}$ when $h$ increases from 20 to $150 \mathrm{~nm}$. This relative shift is slightly more sensitive to the gold thickness in rings due to larger exposed surface to volume ratio (increasing monotonously from $1.7 \%$ to $3.2 \%$ for $h$ increasing from 20 to $150 \mathrm{~nm}$ ). The data presented in Figs. 6 and 7 show that the relative tuning from the rings configuration is larger if compared with the disks one, though resonances occur at longer wavelengths. To emphasize the effect of the optical pump, we report the detail of the tuning of the spectral response versus the pump power value when $h=20 \mathrm{~nm}, a=0$ and $a=250 \mathrm{~nm}$, as reported in Figs. $\underline{8}$ and 9 , respectively.

For the disks, starting from the case of no optical pumping, the resonant wavelength shift develops from 1767.20 to $1808.00 \mathrm{~nm}$ when the pump is set to $40 \mathrm{~mW} / \mathrm{cm}^{2}$.

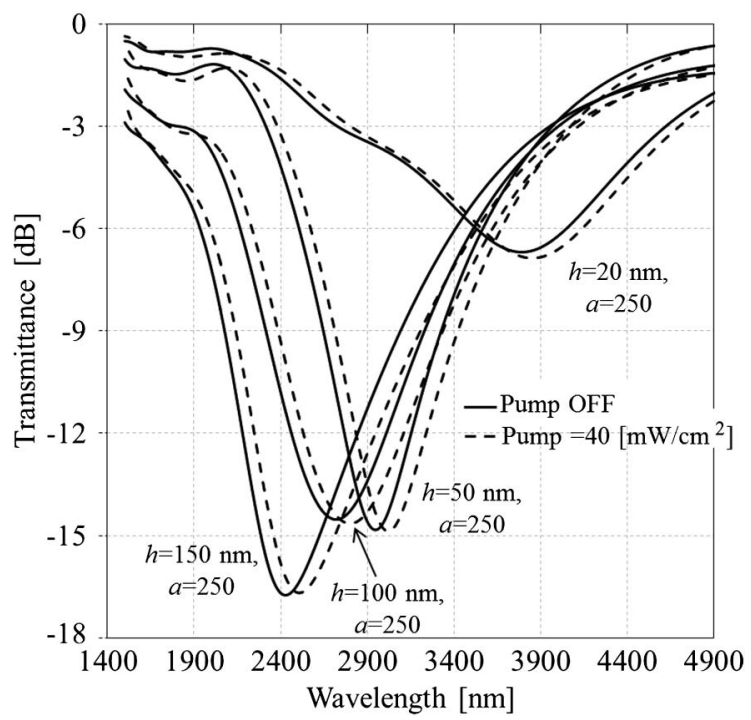

Fig. 7. Simulated zero-order transmittance spectra as a function of the gold thickness $(h)$. The geometry considered is $a=250 \mathrm{~nm}$ (ring), $b=300 \mathrm{~nm}$, and $T=800 \mathrm{~nm}$. The dashed and solid lines compare optical pumping with $P_{\text {sat }}=40 \mathrm{~mW} / \mathrm{cm}^{2}$ to the case of no pumping. 


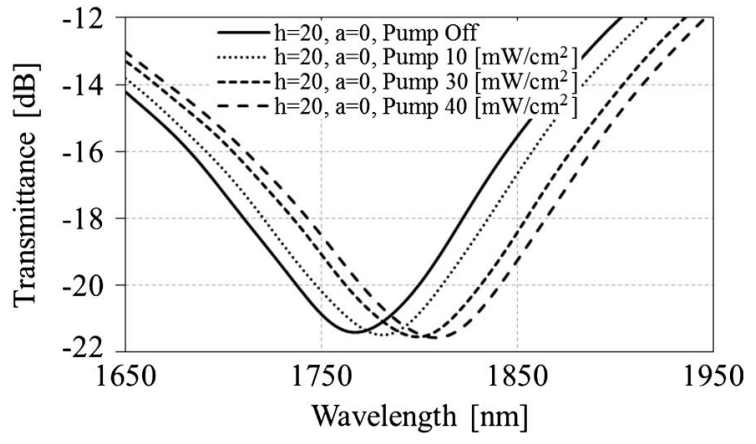

Fig. 8. Detailed tuning of the spectral response due to the laser pump power variation. The geometry considered is $a=0 \mathrm{~nm}, b=300 \mathrm{~nm}$, $h=20 \mathrm{~nm}$, and $T=800 \mathrm{~nm}$.

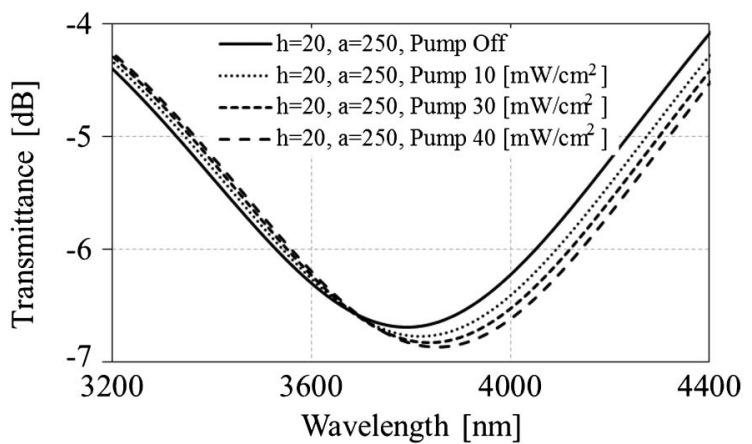

Fig. 9. Detailed tuning of the spectral response due to the laser pump power variation. The geometry considered is $a=250 \mathrm{~nm}$, $b=300 \mathrm{~nm}, h=20 \mathrm{~nm}$, and $T=800 \mathrm{~nm}$.

For the rings, starting from the case of no optical pumping, the resonant wavelength shift develops from 3787 to $3850 \mathrm{~nm}$ when the pump is set to $40 \mathrm{~mW} / \mathrm{cm}^{2}$.

\section{CONCLUSION}

In our numerical studies we have demonstrated the potential for all optical tuning of the plasmon resonance in a periodic metallic structure. The tuning occurs when an optical pump light in the visible spectrum hits the mixture of azo-dye-doped liquid crystal. Significant differences are observed for disks and rings. The proposed device allows precise control over the spectral response and is completely reversible. Compared with conventional (thermally or electrically controlled) LCbased devices, this approach offers new advantages, such as very low power consumption and short response time for the next generation of optical tunable plasmonic devices. Once the geometrical structure is defined, the amount of the wavelength shift depends on the power density of the optical pump. We envisage that the proposed structured thin film may be applied to create tunable mirrors for laser devices and other components. Moreover, the suppressed transmission results in enhanced absorption, which is potentially interesting in the context of energy harvesting for solar cells and photocatalysis.

\section{REFERENCES}

1. T. W. Ebbsen, H. J. Lezec, H. F. Ghaemi, T. Thio, and P. A. Wolff, "Extraordinary optical transmission through sub-wavelength hole arrays," Nature 391, 548-556 (2003).
2. J. A. Porto, F. J. Garcia-Vidal, and P. B. Pendry, "Transmission resonances on metallic gratings with very narrow slits," Phys. Rev. Lett. 83, 2845-2848 (1999).

3. I. S. Spevak, A. Y. Nikitin, E. V. Bezuglyi, A. Levchenko, and A. V. Kats, "Resonantly suppressed transmission and anomalously enhanced light absorption in periodically modulated ultrathin metal films," Phys. Rev. B 79, 161406R (2009).

4. H. Hu, C. Ma, and Z. Liu, "Plasmonic dark field microscopy," Appl. Phys. Lett. 96, 113107 (2010).

5. S. A. Maier, P. G. Kik, H. A. Atwater, S. Meltzer, E. Harel, B. E. Koel, and A. A. G. Requicha, "Local detection of electromagnetic energy transport below the diffraction limit in metal nanoparticle plasmon waveguides," Nat. Mater. 2, 229-232 (2003).

6. N. Yu, J. Fan, Q. J. Wang, C. Pflugl, L. Diehl, T. Edamura, M. Yamanishi, H. Kan, and F. Capasso, "Small-divergence semiconductor lasers by plasmonic collimation," Nat. Photonics 2, 564-570 (2008).

7. S. Olcum, A. Kocabas, G. Ertas, A. Atalar, and A. Aydinli, "Tunable surface plasmon resonance on an elastomeric substrate," Opt. Express 17, 8542-8547 (2009).

8. G. Xu, M. Tazawa, P. Jin, S. Nakao, and K. Yoshimura, "Wavelength tuning of surface plasmon resonance using dielectric layers on silver island films," Appl. Phys. Lett. 82, 3811-3813 (2003).

9. M. Dridi and A. Vial, "Modeling of metallic nanostructures embedded in liquid crystals: application to the tuning of their plasmon resonance," Opt. Lett. 34, 2652-2654 (2009).

10. E. M. Phizicky and S. Fields, "Protein-protein interactions: methods for detection and analysis," Microbiol. Rev. 59, 94-123 (1995).

11. J. C. Yang, J. Ji, M. Hogle, and D. N. Larson, "Metallic nanohole arrays on fluoropolymer substrates as small label-free real-time bioprobes," Nano Lett. 8, 2718-2724 (2008).

12. B. Zhang, Y. Zhao, Q. Hao, B. Kiraly, I. Khoo, S. Chen, and T. J. Huang, "Polarization-independent dual-band infrared perfect absorber based on a metal-dielectric-metal elliptical nanodisk array," Opt. Express 19, 15221-15228 (2011).

13. J. S. T. Smalley, Y. Zhao, A. A. Nawaz, Q. Hao, Y. Ma, I. Khoo, and T. J. Huang, "High contrast modulation of plasmonic signals using nanoscale dual-frequency liquid crystals," Opt. Express 19, 15265-15274 (2011).

14. N. Felidj, J. Aubard, G. Levi, J. R. Krenn, M. Salerno, G. Schider, B. Lamprecht, A. Leitner, and F. R. Aussenegg, "Controlling the optical response of regular arrays of gold particles for surfaceenhanced Raman scattering," Phys. Rev. B 65, 075419 (2002).

15. G. Gilardi, S. Xiao, R. Beccherelli, A. d'Alessandro, and N. A. Mortensen, "Geometrical and fluidic tuning of periodically modulated thin metal films," Photon. Nanostr. Fundam. Appl. 10, 177-182 (2012).

16. S. Xiao and N. A. Mortensen, "Surface-plasmon-polaritoninduced suppressed transmission through ultrathin metal disk arrays," Opt. Lett. 36, 37-39 (2011).

17. D. C. Zografopoulos and R. Beccherelli, "A vertically-coupled liquid-crystal long-range plasmonic optical switch," Appl. Phys. Lett. 102, 101103 (2013).

18. Y. J. Liu, Q. Hao, J. S. T. Smalley, J. Liou, I. C. Khoo, and T. Jun Huang, "A frequency-addressed plasmonic switch based on dual-frequency liquid crystals,” Appl. Phys. Lett. 97, 091101 (2010).

19. Q. Hao, Y. Zhao, B. Krishna Juluri, B. Kiraly, J. Liou, I. C. Khoo, and T. J. Huang, "Frequency-addressed tunable transmission in optically thin metallic nanohole arrays with dual-frequency liquid crystals," J. Appl. Phys. 109, 084340 (2011).

20. D. C. Zografopoulos, R. Beccherelli, A. C. Tasolamprou, and E. E. Kriezis, "Liquid-crystal tunable waveguides for integrated plasmonic components," Photon. Nanostr. Fundam. Appl. 11, 73-84 (2013)

21. D. C. Zografopoulos and R. Beccherelli, "Liquid-crystal tunable metal-insulator-metal plasmonic waveguides and Bragg resonators," J. Opt. 15, 055009 (2013).

22. Y. J. Liu, Y. B. Zheng, J. Liou, I.-K. Chiang, I. C. Khoo, and T. J. Huang, "All-optical modulation of localized surface plasmon coupling in a hybrid system composed of photoswitchable gratings and Au nanodisk arrays," J. Phys. Chem C 115, 7717-7722 (2011) 
23. Y. Zhao, Q. Hao, Y. Ma, M. Lu, B. Zhang, M. Lapsley, I. C. Khoo, and T. J. Huang, "Light-driven tunable dual-band plasmonic absorber using liquid-crystal-coated asymmetric nanodisk array," Appl. Phys. Lett. 100, 053119 (2012).

24. L. De Sio, S. Serak, N. Tabiryan, and C. Umeton, "Mesogenic versus non-mesogenic azo dye confined in a soft-matter template for realization of optically switchable diffraction gratings," J. Mater. Chem. 21, 6811-6814 (2011).

25. G. Gilardi, L. De Sio, R. Beccherelli, R. Asquini, A. d'Alessandro, and C. Umeton, "All-optical and thermal tuning of a Bragg grating based on photosensitive composite structures containing liquid crystals," Mol. Cryst. Liq. Cryst. 558, 64-71 (2012).

26. D. C. Zografopoulos, R. Asquini, E. E. Kriezis, A. d'Alessandro, and R. Beccherelli, "Guided-wave liquid-crystal photonics," Lab Chip 12, 3598-3610 (2012).
27. J. Li, S. Gauza, and S. Wu, "Temperature effect on liquid crystal refractive indices," J. Appl. Phys. 96, 19-24 (2004).

28. http://www.comsol.com.

29. B. Bellini and R. Beccherelli, "Modelling, design and analysis of liquid crystal waveguides in preferentially etched silicon grooves," J. Phys. D 42, 045111 (2009).

30. I.-C. Khoo, Liquid Crystals, 2nd ed. (Wiley, 2007).

31. L. Lucchetti, M. Di Fabrizio, O. Francescangeli, and F. Simoni, "Colossal optical nonlinearity in dye doped liquid crystals," Opt. Commun. 233, 417-424 (2004).

32. G. Gilardi, L. De Sio, R. Beccherelli, R. Asquini, A. d'Alessandro, and C. Umeton, "Observation of tunable optical filtering in photosensitive composite structure containing liquid crystals," Opt. Lett 36, 4755-4757 (2011).

33. P. B. Johnson and R. W. Christy, "Optical constants of noble metals,” Phys. Rev. B 6, 4370-4379 (1972). 